\title{
NOVO MODELO EXPERIMENTAL DE GLAUCOMA EM CÃO PARA O ESTUDO DA CICATRIZAÇÃO APÓS CIRURGIA FILTRANTE ASSOCIADA AO USO DE AGENTE ANTIFIBRÓTICO*
}

\section{ANTONIO FELIPE P.F. WOUK ${ }^{1}$; SILVANA CÍRIO² ${ }^{2}$ GIULIANA GELBECK KASECKER ${ }^{3}$; CASSIANA RAMOS ${ }^{2}$; ROSANA KELLER RICHTER ${ }^{2}$}

\author{
${ }^{1}$ Curso de Medicina Veterinária, Universidade Federal do Paraná e Pontifícia Universidade Católica do Paraná; \\ ${ }^{2}$ Curso de Medicina Veterinária, Centro de Ciências Agrárias e Ambientais, Pontifícia Universidade Católica do \\ Paraná; ${ }^{3}$ Mestranda, Curso de Pós-Graduação em Ciências Veterinárias, Universidade Federal do Paraná.
}

\begin{abstract}
Experimental glaucoma was produced in all the eyes of three dogs by means of intracameral injections of physiological solution saturated with graphite microparticles. Homogeneous absolute glaucomas were then obtained by means of this technique. They were evidenced eight weeks after the intracameral injections. Following this experimental model, glaucomas were obtained in other 9 dogs. Each animal was subjected to two types of surgery, that is, filtration trabeculectomy and iridencleisis and by two antifibrotic topical treatments during the surgery with Mitomicin $\mathrm{C}$ and acetylsalicylic acid. Clinical and histopathologic evaluation of the experimental units were performed with emphasis in the third, sixth and twelve months after surgery. Iridencleise showed to be the most efficient surgical procedure in spite of being associated to the antifibrotic treatment. Acetylsalicylic acid displayed better antifibrotic activity than Mytomicin $\mathrm{C}$ showing no collateral effects as the formation of hypotonic globes for instance. This new glaucoma model may also be used for other animal species. Besides being simple and inexpensive it acts as a graphite histologic "marker" of the aqueous humor "path". Moreover, it allows to be used in the dog in pharmacological tests as well as in other protocols of glaucoma filtration surgery, specially in studies regarding the use of acetylsalicylic acid as eye antifibrotic agent.
\end{abstract}

RESUMO - Inicialmente três cães (6 olhos) foram submetidos ã injeções intracamerais de uma solução fisiológica saturada com micropartículas de grafite buscando-se a obtenção do glaucoma experimental. Ao final de 8 semanas constatou-se, de forma homogênea, glaucomas absolutos em todos os olhos. Na seqüência produziu-se, segundo este modelo, glaucoma em outros 9 cães (18 olhos), cada animal sendo submetido a dois tipos de cirurgia filtrantetrabeculectomia e iridencleise- e a dois tratamentos transoperatórios antifibróticos Mitomicina C e ácido acetil salicílico. As unidades experimentais assim constituídas foram estudadas clinicamente e histopatologicamente, com evidências aos 3, 6 e 12 meses de pós-operatório. O procedimento cirúrgico mais eficaz, independente do tratamento antifibrótico associado, foi a iridencleise, evidenciado por uma bolha de filtração patente um ano após a intervenção. $\mathrm{O}$ ácido acetil salicílico mostrou resultados antifibróticos superiores à Mitomicina $\mathrm{C}$ e ao contrário desta última, sem apresentar efeitos colaterais como globos hipotônicos. É lícito propor este novo modelo experimental de glaucoma para outras espécies por ser simples, barato, além de atuar como um "marcador" histológico do "caminho" do aquoso. Além disto poderá ser novamente empregado no cão visando testar outras técnicas de cirurgia filtrante e outros protocolos farmacológicos do glaucoma mas para sobretudo consolidar os estudos do uso do Ácido Acetil Salicílico como agente antifibrótico no olho.

\section{Introdução}

O glaucoma é uma elevação da pressão intraocular (PIO) suficiente para lesar o nervo óptico e causar cegueira temporária ou permanente. Por isto, é considerado uma emergência ocular, onde o objetivo maior de toda terapia é abortar o dano ao nervo óptico reduzindo a PIO. Embora alguns casos de glaucoma possam ser manejados medicamente, na maioria deles o tratamento definitivo é cirúrgico (BIRNBAC e LEEN, 1996; GELATT, 1991;

* Com auxílio do Conselho Nacional do Desenvolvimento Científico e Tecnológico - CNPq, Processo \# 301457/85-9 (RN).
GIONFRIDDO, 1995; SLATTER, 1990; WIL COCK et al., 1990; WIIKIE, 1994).

Um modelo experimental de glaucoma permite testar e desenvolver novas medicações, testar procedimentos cirúrgicos conhecidos e propor técnicas inovadoras na abordagem do tratamento. Busca-se modelos animais adequados a esta finalidade (LEE et al., 1987). Os mais recentemente desenvolvidos foram o de fotocoagulação a "laser" de argônio da trama trabecular e mediante injeção de microesferas de látex na câmara anterior de olhos de macacos (WEBER e ZELENAK, 1996).

$\mathrm{O}$ animal doméstico onde se conhece melhor a fisiologia do humor aquoso e por conseguinte o sistema de pressão intra-ocular é o cão. Neles a PIO varia de 15 a $25 \mathrm{mmHg}$ e de 85 a $95 \%$ do humor 
aquoso deixa o olho através da chamada rota de drenagem convencional. Nesta rota, o humor aquoso passa entre os ligamentos pectinados e chega ao interior dos espaços extratrabeculares da trama trabecular da úvea anterior e daí, para os espaços da trama trabecular córneo-escleral pertencente ao ângulo irido-trabeculo-corneano (ângulo camerular ou de drenagem). O restante é drenado pelo trato úveo-escleral, rota não convencional. A obstrução da livre circulação do humor aquoso causa aumento da PIO (GIONFRIDDO, 1995).

Quando pesquisávamos a cicatrização de tendões submetidos a implante com fibra de carbono em eqüinos e cães, ocorreu-nos a hipótese de que micropartículas destas fibras injetadas na câmara anterior poderiam causar a obliteração da trama trabecular e causar aumento da PIO. Dentre as espécies domésticas, o eqüino é aquela onde o glaucoma é mais raro, provavelmente devido a uma maior facilidade de circulação do humor aquoso. Para um teste pré-experimental mais crítico escolhemos três cavalos que sofreram a injeção, com uma agulha $30 \times 8$, na câmara anterior de um dos olhos, de 1,0 ml de uma solução saturada de micropartículas de fibra de carbono em soro fisiológico; o olho adelfo permaneceu como controle. Os animais foram previamente sedados com detomidina. Em 24 horas, obteve-se megaloftalmia por aumento da PIO com todos os sinais clínicos de glaucoma hiperagudo com uveíte. Começou aí nosso novo modelo experimental de glaucoma.

Em animais, o glaucoma é mais comum em cães e, freqüentemente, é de difícil controle, mesmo cirurgicamente, pois a melhor estratégia cirúrgica ainda não foi encontrada. A cirurgia deve reduzir a PIO sem destruir a visão remanescente. Dentre as técnicas mais utilizadas estão as cirurgias filtrantes como a ciclodiálise e a trabeculectomia, as quais têm sucesso a curto termo mas, com três a seis meses de evolução, tem o orifício de drenagem obliterado por uma cicatriz e, com isto, a recorrência do glaucoma (GIONFRIDDO, 1995). Por esta razão, julgamos ser o cão, o modelo animal crítico para o estudo de cirurgias filtrantes no tratamento do glaucoma.

A cicatrização de feridas é um fenômeno complexo, natural e normal, limitado pelo tempo e essencial para a sobrevivência de todos os organismos vivos. Um agente comum ao processo de cicatrização é o fibroblasto, responsável pela maior parte da formação do tecido cicatricial (LEE, 1996).

Os processos de cicatrização e formação de cicatriz têm influência na patogênese ou na falha de tratamento em, virtualmente, todas as principais doenças que cegam atualmente no mundo. Em particular, o processo de cicatrização após cirurgia filtrante do glaucoma é o principal determinante da pressão intra-ocular final (KHAW, 1996).

A inflamação aguda acompanhada de fibrose e neovascularização são sérias complicações do tratamento cirúrgico do glaucoma. Cada vez mais dados sugerem que tais complicações são mediadas por prostaglandinas encontradas em quantidades aumentadas no humor aquoso de pacientes humanos com glaucoma. O Fator de Crescimento Básico do Fibroblasto (BFGF) e o Fator de Transformação de Crescimento (3 (TGF (3) modulam a síntese e a liberação de prostaglandinas e podem estar envolvidos na etiologia da fibrose inflamatória intra-ocular e na neovascularização (GODDARD, 1996). Neste sentido, ao nosso ver, seria interessante verificar a ação do ácido acetil salicílico, reconhecidamente um excelente antiprostaglandina, como facilitador da potência da cirurgia filtrante.

A mitomicina é um agente alquilante que inibe a proliferação de fibroblastos e é de 100 a 300 vezes mais potente que o 5-fluorouracil. Este é o mecanismo pelo qual ela pode melhorar a evolução de cirurgias filtrantes do glaucoma (SKUTA, 1996). Nestas situações, ela permitiu a patência das bolhas de filtração utilizada no período intraoperatório (ZIEL e JOHNSTONE, 1996; SANDERS et al., 1996; IGARASHI et al., 1996) ou pós-operatório (HYUNG et al., 1996; MINCKLER, 1996; SYGIEL et al., 1996). A mitomicina parece possuir um efeito tóxico sobre as células endoteliais vasculares e sobre o corpo ciliar. $\mathrm{O}$ primeiro efeito parece ser o responsável pela falta de vascularização observada freqüentemente no sítio cirúrgico. As alterações no corpo ciliar contribuiriam para a prolongada hipotonia pósoperatória que por vezes ocorre no homem submetido à trabeculectomia associada ao uso de mitomicina. Com relação a estes e outros agentes inibidores da cicatrização, as pesquisas atuais e futuras devem ser dirigidas ao estudo dos efeitos a curto e a longo prazo destes sobre o olho e também à procura de um agente com uma relação riscobeneficio potencialmente melhor (SKUTA, 1996). Novamente aqui levantamos a hipótese do uso do ácido acetil salicílico, também conhecido pela sua ação queratolítica e pouco risco tóxico.

Embora as culturas celulares e os estudos clínicos tragam-nos informações que podem melhorar nossos tratamentos para cicatrizes, os modelos animais possibilitam informações vitais que não podem ser obtidas facilmente de outra maneira. Isto ainda, a despeito das diferenças na cicatrização da ferida entre os modelos, especificamente, a magnitude da resposta cicatricial.

Os modelos animais de cirurgia filtrante do 
Novo Modelo Experimental de Glaucoma em Cão

glaucoma têm nos ajudado e vão nos ajudar a:

- Testar os tratamentos disponíveis para cicatrizes;

- Entender a biologia básica da cicatrização após cirurgia, especialmente as complexas interações que ocorrem em uma ferida "in vivo", o que não pode ser reproduzido em cultura celular;

- Compreender os efeitos focais mensuráveis de vários anti-metabólitos e outros antifibróticos em aplicação única nas populações celulares locais e transpor estes princípios para a prática clínica;

- Prevenir os efeitos colaterais destes tratamentos a fim de modular e controlar o processo de cicatrização no olho (KHAW, 1996).

Diante do exposto, são os seguintes os objetivos deste trabalho:

- definir e padronizar um novo modelo de glaucoma experimental em cães;

- avaliar clinicamente a longo prazo duas técnicas de cirurgia filtrante associadas ao uso de dois agentes antifibróticos;

- estudar histologicamente a evolução cicatricial destas estratégias terapêuticas.

\section{Material e Métodos. Resultados}

Foram utilizados doze cães sadios, sem raça definida, com peso médio de $12 \mathrm{~kg}$, provenientes do Serviço de Busca e Apreensão da Prefeitura Municipal de Curitiba, os quais foram mantidos em canis individuais com solário e alimentados com ração comercial para cães.

Procedimentos para a produção do glaucoma experimental. Foram utilizados três cães (6 olhos) para a produção do glaucoma experimental. Realizou-se a Curva Pressórica Basal, a fim de conhecer a pressão intra-ocular (PIO) normal dos cães. Durante três dias, a cada hora, por um período diário de 6 horas, foi realizada a leitura da PIO nos dois olhos de cada animal, totalizando 36 medidas. Dois métodos tonométricos foram utilizados, sendo um em cada olho, determinado por sorteio. Os métodos empregados foram o de identação (tonômetro de Schiotz) e aplanação (TonoPen). Obteve-se para as 18 medidas obtidas por tonometria de aplanação diretamente a média de $18,6 \mathrm{mmHg}$ (14 a $16 \mathrm{mmHg}$ ) com desvio padrão de 2,9. Para as medidas com o Tonômetro de Schiotz, empregou-se o peso de 7,5g e a média dos valores obtidos foi 7 ( 5 a 9), o que corresponde na escala de Pfeiffer a uma pressão média de $22 \mathrm{mmHg}$.

Uma solução saturada de grafite foi preparada por meio da mistura de grafite em pó (lubrificante comercial) com granulometria conhecida de 5 micrômetros, esterilizado em óxido de etileno com soro fisiológico (cloreto de sódio a $0,9 \%$ ).

Os animais foram sedados por meio de aplicação intramuscular de cloridrato de xilazina (Rompum $\left.{ }^{\circledR}\right)$, seguida de anestesia ocular tópica com colírio de proparacaína (Anestalcon $\left.{ }^{\circledR}\right)$. Após estes procedimentos, aspirou-se, em uma seringa de insulina, um volume padrão de $0,25 \mathrm{ml}$ da solução saturada de grafite que foi injetada na câmara anterior dos 6 olhos, através de paracentese em córnea clara, posição límbica de 12 horas.

$\mathrm{Na}$ seqüência do procedimento, as PIO foram inicialmente controladas a cada hora, durante seis horas, mediante tonometria de aplanação, no que pôde ser observada, até a terceira hora, uma diminuição de $4 \mathrm{mmHg}$ em média da PIO em relação aos valores basais. Até a sexta hora, à exceção de um olho, as PIO se elevaram para atingir os valores basais.

Tabela 1. Evolução cronológica em horas dos achados clínicos após a seqüência de aplicações de solução saturada de grafite na câmara anterior dos olhos dos cães.

\begin{tabular}{l|l}
\hline \multicolumn{1}{c|}{ Tempo de Evolução } & \multicolumn{1}{c}{ Achados } \\
\hline 6 horas & $\begin{array}{l}\text { Fotofobia, blefarospano, ângulo camerular aberto com ausência de deposição do } \\
\text { grafite. }\end{array}$ \\
\hline 12 horas & $\begin{array}{l}\text { Conjuntivite, irido-ciclite com Tyndall positivo, ângulo camerular aberto com discreta } \\
\text { deposição do grafite. }\end{array}$ \\
\hline 24 horas/2a aplicação & Blefarospano e fotofobia mais intenso. \\
\hline 36 horas & Ângulo camerular semi-aberto com moderada impregnação pelo grafite. \\
\hline 60 horas/3 $/ 3^{a}$ aplicação & Acentuada conjuntivite e irido-ciclite. \\
\hline 72 horas & $\begin{array}{l}\text { Início de modificações do fundo, afinamento de artérias retinianas, ângulo semi- } \\
\text { fechado, acentuada impregnação pelo grafite. }\end{array}$ \\
\hline & $\begin{array}{l}\text { Ângulo camerular fechado com acentuada deposição de grafite e PIO superior a 40 } \\
\text { mmHg. }\end{array}$ \\
\hline
\end{tabular}

*Um olho apenas, por não apresentar os sinais relatados acima após as 48 horas, sofreu uma Quarta aplicação com 72 horas de evolução, resultando em ângulo fechado devido à acentuada impregnação pelo grafite e PIO superior a $30 \mathrm{mmHg}$.

Daí em diante, mediu-se a PIO a cada 12 horas até o terceiro dia e na seqüência diariamente, até a obtenção de um quadro de hipertensão ocular estável por cinco dias. Na ausência de hipertensão ocular 24 horas após a primeira injeção intra-cameral, esta era repetida a cada 24 horas até a obtenção do quadro 
estável de hipertensão. Com exceção de um olho que necessitou de uma quarta injeção, os outros cinco olhos desenvolveram hipertensão após três injeções. Com este protocolo, os 6 olhos revelaram PIO estáveis superiores a $30 \mathrm{mmHg}$, com variação de 31 a $42 \mathrm{mmHg}$ e média de $36 \mathrm{mmHg}$. Nas 8 semanas seguintes observou-se um declínio gradativo da PIO, porém permanecendo com valor médio ao final da oitava semana de $31 \mathrm{mmHg}$ para os 6 olhos.

Os testes estatísticos empregados para análise comparativa das médias das PIO (análise de variância, Método de Newman-Keuls, Duncan e Tukey e Teste do "T" de Student) revelaram uma diferença significativa ao nível de $5 \%$ em relação às PIO basais. A significativa hipertensão ocular obtida confirma o sucesso do novo modelo experimental de glaucoma.

Avaliação clínica do glaucoma experimental. Paralelamente à medida das pressões intra-oculares (PIO), nos mesmos períodos anteriormente citados, realizou-se exame físico oftálmico, oftalmoscopia direta e indireta e gonioscopia mediante o uso de uma lente de Barkan. Este exame avaliou o grau de patência do ângulo irido-trabéculo-corneano (aberto, semi-aberto, semi-fechado ou fechado) e o grau de deposição das partículas de grafite no ângulo (ausente, discreto, moderado e acentuado). Para fins estatísticos, estes parâmetros receberam valores de zero a três, sendo considerado três um ângulo fechado, com grau de deposição das partículas de grafite acentuado.

Tabela 2. Evolução cronológica em semanas das alterações fundoscópicas observadas após a indução do glaucoma experimental.

\begin{tabular}{l|l}
\hline \multicolumn{1}{c|}{ Tempo de Evolução } & \multicolumn{1}{c}{ Achados } \\
\hline 1 semana & $\begin{array}{l}\text { Diminuição do calibre das artérias retinianas, palidez do disco, início do alargamento } \\
\text { concêntrico da escavação do disco óptico. }\end{array}$ \\
\hline 2 a 3 semanas & $\begin{array}{l}\text { Desenvolvimento de fosseta do nervo óptico por ectasia posterior localizada da lâmina } \\
\text { cribiforme. }\end{array}$ \\
\hline 4 semanas & $\begin{array}{l}\text { Cabeças dos nervos ópticos escavadas e com sinais de atrofia com modificação da cor } \\
\text { para o cinza e aparecimento de vasos retinianos em forma de chama na região peri- } \\
\text { valvar. }\end{array}$ \\
\hline 8 semanas & Cegueira bilateral. Glaucoma clínico de ângulo fechado com PIO média de 31 mmHg. \\
\hline 48 horas $/ 3^{\text {a }}$ aplicação & Acentuada conjuntivite e irido-ciclite. \\
\hline
\end{tabular}

Ao longo das oito semanas de observação, o que se verificou clinicamente foi uma redução gradativa dos sinais inflamatórios e o início das alterações degenerativas no segmento anterior e posterior dos olhos. Um quadro de uveíte hipertensiva concomitante ao glaucoma de ângulo fechado persistiu até a sexta semana.

Em nenhum momento verificou-se um quadro de uveíte posterior. Os sinais de atrofia retiniana na zona tapetal tiveram início na quinta semana em 4 olhos de dois animais, com lesões hiper-relletivas inicialmente peripapilares e depois periféricas na forma de praias não coalescentes. Dois olhos de um animal não chegaram a apresentar sinais de atrofia retiniana até $o$ final da oitava semana de observação.

As alterações do segmento anterior observadas conforme a evolução do quadro incluíram congestão episcleral, endotelite com discreto edema de córnea (não impedindo a visualização do fundo), midríase fixa e persistente, megaloftalmia, desvio temporal do globo (em dois olhos do mesmo animal), estrias da membrana de Descemet (em quatro olhos de dois animais) e ceratite com "pannus" em todos os olhos.

No final da oitava semana, todos os animais apresentaram cegueira bilateral, com glaucoma clínico de ângulo fechado (PIO média de 31
mmHg). Neste momento foram submetidos a eutanásia, tendo seus olhos removidos e submetidos a exame histopatológico. Os achados mais relevantes foram a perda de células ganglionares e axônios do nervo óptico, ectasia da lâmina crivosa da esclera e degeneração com perdas celulares das camadas plexiforme externa e interna da retina.

Cirurgias filtrantes e tratamentos anti-fibróticos. Foram utilizados 9 cães (18 olhos), nos quais se testou duas técnicas cirúrgicas filtrantes iridencleise e trabeculectomia, e dois tratamentos anti-fibróticos - MitomicinaC (M-c) e ácido acetil salicílico (AAS). Os dois procedimentos e os dois tratamentos foram testados em cada animal, sendo 0 olho decidido por sorteio. Desta forma, cada animal constituiu uma unidade experimental.

Inicialmente, reproduziu-se nestes cães o modelo experimental de glaucoma anteriormente descrito e testado. Com 5 dias de hipertensão estável, superior a $40 \mathrm{mmHg}$, ângulo camerular fechado e acentuada impregnação da fenda ciliar pela solução saturada de grafite, todos os 18 olhos, apresentando ainda quadro de uveíte hipertensiva, foram operados. Foram realizadas 9 trabeculectomias e 9 iridencleises, metade dos procedimento tratados com AAS e metade com M-c. As técnicas cirúrgicas realizadas foram aquelas conhecidas na literatura e já consolidadas 
para o cão. A anestesia foi geral gasosa com éter em circuito semi-fechado.

A M-c, na concentração de $0,4 \mathrm{mg} / \mathrm{ml}$, foi aplicada sobre o sítio operatório durante a cirurgia por 7 minutos com o auxílio de uma microesponja oftálmica. A instilação de M-c no pós-operatório, inicialmente prevista para 7 dias, quatro vezes ao dia, foi suspensa ao final do segundo dia devido à grande quemose provocada.

O AAS foi empregado no sítio operatório durante a cirurgia sob a forma de grânulos na dose total de $100 \mathrm{mg}$, antes da realização da sutura da conjuntiva bulbar, não sendo utilizado no pós- operatório. Os animais não apresentaram complicações relacionadas ao seu uso.

Os mesmos parâmetros e a mesma metodologia instrumental e não instrumental de exames descrita anteriormente voltaram a ser empregados nesta fase. Adicionalmente, avaliou-se a patência da "bolha" de filtração cirúrgica segundo a seguinte graduação: zero para ausência de "bolha" de filtração, 1 para pequena filtração e 2 para filtração adequada. Os animais foram avaliados diariamente durante o primeiro mês e a partir daí, semanalmente.

Tabela 3. Freqüência e tipo de alterações clínicas observadas após o uso tópico de Mitomicina-C em olhos de cães com glaucoma experimental.

\begin{tabular}{l|c}
\multicolumn{1}{c|}{ Alterações } & $\begin{array}{c}\text { Número de olhos que } \\
\text { apresentaram alteração }\end{array}$ \\
\hline Irritação do epitélio corneano & 4 \\
\hline Hipotonia pós-operatória importante & 5 \\
\hline Descolamento da coróide & 3 \\
\hline
\end{tabular}

Em todos os animais o ato cirúrgico agravou o quadro de uveíte, o que veio a desaparecer em torno do final da terceira semana de pósoperatório, à exceção de dois olhos tratados com M-c, pertencentes a dois animais. Estes olhos, devido à hipotonia, fizeram câmara rasa, sinéquia posterior, iris "bombé" e uveíte hipertensiva, evoluindo, na seqüência, para "pthisis bulbi". Observou-se também, descolamento da coróide e irritação corneana, com ausência de "bolha" de filtração (grau zero), aos 3 meses. Os outros três olhos, dos mesmos animais, tratados com AAS, apresentavam neste momento "bolha" de filtração adequada. Estes dois cães foram então submetidos a eutanásia e tiveram seus olhos enucleados e submetidos a exame histopatológico, constituindo-se no primeiro estudo, com três meses de evolução.

Os outros seis olhos tratados com AAS seguiram até o final do sexto mês clinicamente calmos e normotensos com "bolha" de filtração adequada (grau 2). Dos outros seis olhos tratados com M-c, apenas um, onde foi realizada uma iridencleise, encontrava-se calmo e normotenso com "bolha" de filtração adequada. Os outros cinco olhos apresentavam complicações: dois, submetidos a trabeculactomia, apresentaram bolha de filtração pequena e três com malácea da esclera e hipotonia ocular importante. Ao final destes 6 meses, um outro grupo de três animais foi submetido à eutanásia e seus olhos foram enviados para estudo histopatológico.

Os olhos dos animais restantes foram avaliados clinicamente até um ano de evolução.

Tabela 4. Sinais clínicos e graduação de filtração do humor aquoso observados um ano após determinada cirurgia filtrante e agente antifibrótico empregado.

\begin{tabular}{l|l}
\hline \multicolumn{1}{c|}{ Agente anti-fibrótico empregado } & \multicolumn{1}{c}{ Sinais observados } \\
\hline Ácido acetil salicílico & "Bolha" adequada (grau 2)- olho calmo-iridenclise \\
& Pequena "bolha" (grau 1)- olho calmo-trabeculectomia \\
\hline Mitomicina-C & $\begin{array}{l}\text { "Bolha" ausente (grau zero), hipertensão ocular discreta, sinais } \\
\text { fundoscópicos de início de escavação e palidez do disco óptico e } \\
\text { afinamento dos vasos retinianos. }\end{array}$ \\
\hline
\end{tabular}

O procedimento cirúrgico mais eficaz, independente do tratamento anti-fibrótico, foi a iridencleise, evidenciado pelas bolhas de filtração, ao contrário da trabeculectomia, que com seis meses de evolução, já não permitia filtração tão satisfatória. De fato, a interposição de tecido iriano ao orifício escleral e por sobre ele tende a minimizar o fenômeno cicatricial. Já a trabeculectomia, originalmente produz uma fistula menor, que se comunica com o espaço subescleral favorecendo a fibroplasia e a formação de uma cicatriz selante.

$\mathrm{O}$ agente anti-fibrótico clinicamente mais eficaz foi o AAS, pois permitiu durante todo o tempo boa filtração. O uso da M-c apresentou graves complicações que culminaram com olhos hipotônicos ou bolhas sem filtração adequada, com ambas técnicas cirúrgicas.

Estudo histopatológico. Para o estudo histopatológico, foram constituídos mediante sorteio três grupos de três animais. Aos 3, 6 e 12 
meses de evolução pós-operatória, foi sacrificado um grupo de cães. Os olhos foram fixados em líquido de Bouin e endurecidos em álcool etílico. Foram cortados de forma a se obter uma "calota" sagital incluindo córnea, úvea anterior, córioretina e nervo óptico. Foram corados pelas técnicas de Hematoxilina-Eosina , Tricromo de Mallory, Azul de Toluidina e Tricromo de Schorr. A histopatologia confirmou os achados clínicos. As alterações histológicas do disco e cório-retina nos dois olhos hipertensos ao final do primeiro ano foram também de perda celular ganglionar na cabeça do disco óptico e nas camadas plexiforme externa e interna da retina, ectasia discreta da lâmina crivosa da ecesclera e pequena escavação concêntrica da cabeça do disco, com afinamento das camadas histológicas destas estruturas.

Nos locais das "bolhas" de filtração e na sua periferia imediata, as cicatrizes se mostraram histologicamente com menor fibroplasia nas situações onde foi empregado 0 AAS, comprovando uma ação anti-fibrótica mais importante. Com o uso da M-c, observou-se fibroplasia mais intensa e, com um ano de evolução, uma cicatriz fibrótica obliterando quase a totalidade da fistula estabelecida cirurgicamente.

Com relação à histologia do ângulo iridotrabeculo-corneano (fenda ciliar) e espaço supraciliar, as partículas de grafite acabaram por produzir o colapso da fenda ciliar e não foram além do espaço supra-ciliar, não atingindo a cório-retina. O espaço supra-ciliar do cão revelou-se pequeno. As partículas de grafite não penetraram a rede trabecular córneo escleral interna.

\section{Discussão}

O modelo experimental de glaucoma utilizando o grafite em pó mostrou-se eficiente, produzindo o desejado colapso da fenda ciliar, mimetizando o glaucoma de ângulo fechado. Trata-se portanto de uma novo modelo para o estudo da doença, o que deve ser buscado de acordo com LEE et al. ( 1987) e WEBER e ZELENAK ( 1986).

A injeção na câmara anterior destas partículas, causou por um tempo, especialmente nas fases iniciais, uma uveíte anterior asséptica, de fixndo mecânico e antigênico. A reação inflamatória decresceu ao longo do experimento para no final não existir de forma ativa. Pode-se dizer que no início o modelo cria um glaucoma de ângulo fechado com uveíte anterior hipertensiva.

Devido ao tamanho das partículas, estas jamais chegaram ao espaço coroidal e, portanto, em nenhum momento verificou-se uma panuveíte com cório-retinite. Histologicamente, devido à cor negra das partículas de grafite, a marcação dos tecidos pela mesma tornou-se bem evidente, permitindo desta forma " mapear" a via de drenagem do humor aquoso, um aspecto bastante interessante do modelo.

O protocolo de acompanhamento clínico estabelecido para o modelo permitiu a descrição cronológica das alterações do olho sob hipertensão $\mathrm{e}$ isto constitui-se em elemento absolutamente original e de grande valia para $o$ melhor conhecimento do quadro clínico do glaucoma em cães, sobretudo pelo reconhecimento das alterações iniciais do mesmo pois são estas que permitem o diagnóstico precoce.

Com relação às duas técnicas cirúrgicas, ficou patente que a iridencleise produz uma fistula de drenagem maior e mais eficiente pois "resiste" melhor à fibroplasia cicatricial, a qual tende a obliterar, especialmente no cão, as cirurgias filtrantes para o glaucoma, o que vai de encontro ao estabelecido por diferentes autores (GELATT, 1991; GIONFRIDO, 1995; GODDARD, 1996; KHAW, 1996; LEE, 1996; WILCOCK et al., 1990; WILKIE, 1994)

No que diz respeito ao tratamento anti-fibrótico, clinicamente e histologicamente o Ácido Acetil Salicílico, mesmo empregado apenas durante a cirurgia, mostrou-se superior determinando a patência da bolha de filtração, ao contrário da Mitomicina-C. Esta última, devido à série de efeitos colaterais que produziu, a exemplo do que pode também ocorrer em seres humanos (IGARASHI et al., 1996; MINCKLER, 1996; SANDERS et al., 1996; SKUTA, 1996; SYGIEL et al., 1996; ZIEL e JOHNSTONE, 1996) pode estar contra-indicado o seu uso em cães, como agente antifibrótico na cirurgia filtrante do glaucoma.

$\mathrm{O}$ conjunto dos resultados demonstram a possibilidade de se manter uma cirurgia filtrante patente no cão, por um período longo de um ano, quando se produz uma fistula mais ampla que se comunica diretamente com e espaço subconjuntival bulbar associado ao uso tópico de AAS como agente anti-fibrótico.

Vale salientar que a decisão de se empregar o AAS como agente anti-fibrótico no olho é original e os resultados são muito animadores. Com base nisto, serão realizados outros estudos, testando outras formulações, vias de administração e protocolos terapêuticos.

O cão é um modelo crítico de teste para cirurgias filtrantes do glaucoma, pela importância da fibroplasia que facilmente se instala e assim promove do fechamento das fístulas cirúrgicas. Por esta razão, os resultados aqui obtidos poderão servir à pesquisa do glaucoma na espécie humana, atualmente $\mathrm{o}$ assunto mais pesquisado pela oftalmologia no mundo.

É lícito propor este novo modelo experimental 
de glaucoma para outras espécies animais como também voltar a empregá-lo no cão visando testar outras técnicas cirúrgicas e outros tratamentos médicos, bem como para consolidar o estudo do ácido acetil salicílico como agente antifibrótico no olho.

\section{REFERÊNCIAS BIBLIOGRÁFICAS}

BIRNBAC, C.D.; LEEN, M.M. Digital assesment of intraocular pressure. Investigative Ophtalmology \& Visual Science. Philadelphia, PA, 37(3), s-814, fev. 15, 1996.

GELATT, K. N. The canine glaucomas. In: Veterinary Ophtalmology. 2. ed. Philadelphia, PA: Lea \& Febiger, 1991. p. 396-428.

GIONFRIDDO, J. R. Recognizing and managing acute and chronic cases of glaucoma. Symposium on red eye in small animals. Veterinary Medicine. Lenexa, KS, p. 26 5-275, mar. 1995.

GODDARD, D. Mechanisms of inflamatory fibrosis in glaucoma. Investigative Ophtalmology \& Visual Science. Philadelphia, PA, 37(3), s-423, fev. 15, 1996.

HYUNG, S.M. ; CHOI, M.Y.; JEAN, W.C. Effects of postoperative mitomycin $\mathrm{C}$ eyedrop on trabeculectomy in refractory glaucoma patients. Invetigative Ophtalmology \& Visual Science. Philadelphia, PA, 37(3), s-24, fev. 15, 1996.

IGARASHI, S.; SIMMONS, R.B.; IGARASHI, H.; MONTENEGRO, M.H. KASAHARA, N.; YOSHIDA, A.; SIMMONS, R. J. Use of trans conjunctival mitomycin $\mathrm{C}$ for internal revision of glaucoma filtration surgery. Investigative Ophtalmology \& Visual Science. Philadelphia, PA, 37(3), s-423, fev. 15, 1996.

KHAW, P.T. Animal models of wound healing: lessons from the laboratory to the clinic. Investigative Ophtalmology \& Visual Science. Philadelphia, PA, 37:3, s-422, fev.15, 1996.

LEE, D. A. Animal models and cellular studies of wound healing. Investigative Ophtalmology \& Visuial Science. Philadelphia, PA, 37:3, s-422, fev.15, 1996.
LEE, P.; PODOS, S.M.; SERLE, J.B.; CAMRAS, C.B.; SEVERIN, C.H. Intraocular pressure effects of multiple doses of drugs applied to glaucomatous monkey eyes. Archives of Ophtalmology. 105:249252, fev. 1987.

MINCKLER, D. Antifibrosis agents in drainage implant surgery. Investigative Ophtalmology \& Visual Science. Philadelphia, PA, 37(3), s-423, fev.15, 1996.

SANDERS, S.P.; CANTOR, L.B. ; DOBLER, A.A.; HOOP, J.S.; SPONSEL, W.E. Mitomycin $\mathrm{C}$ in complicated trabeculectomy: a prospective comparison of 0,2 to $0,4 \mathrm{mg} / \mathrm{cc}$. Investigative Ophtalmology \& Visual Science. Philadelphia, PA, 37(3), s-25, fev.15, 1996.

SKUTA, G.L. Adjunctive mitomycin in glaücoma filtering surgery. Investigative Ophtalmology \& Visual Science. Philadelphia, PA, 37(3), s-422, fev.15, 1996.

SLATTER, D.H. Glaucoma. In: Fundamentals of Veterinary Ophtalmology. 2. ed. Philadelphia, PA : W. B. Saunders Company, p. 338-364, 1990.

SYGIEL, B.R.; SHIN, D.H.; JOHNSTON, E.; JUZYCH, M.S.; KUPIN, T.; OLIVER, M.G. M. The role of adjunct mitomycin $\mathrm{C}$ in primary trabeculectomy with and without risk factors. Investigative Ophtalmology \& Visual Science. Philadelphia, PA, 37(3), s-25, fev.15, 1996.

WEBER, A. J.; ZELENAK, D. Experimental glaucomamddel in the prim te eye induced by injection of latex microspheres into the anterior chamber. Investigative Ophtalmology \& Visual Science. Philadelphia, PA, 37(3), s-815, fev. 15, 1996.

WILCOCK, B.P.; PEIFFER, R.L.; DAVIDSON, M.G. The causes of glaucoma in cats. Veterinary Pathology. 27: 35-40, 1990.

WILKIE, D.A. Glaucoma. In: BIRCHARD, S.J.; SHERDING, R.G. Saunders Manual of Small Animal Practice. Philadelphia, PA: W.B. Saundres Company, 1994.

ZIEL, C.J.; JOHNSTONE, M.A. Combined phacoemulsification and trabeculectomy with Mitomicin and postoperative 5-fluoracil (5-FU). Eye Clinic of Wisconsin, Wausau, Wi. Investigative Ophtalmology \& Visual Science, 37(3):15-32, 1996. 